(C) 2014 IEEE. Personal use of this material is permitted. Permission from IEEE must be obtained for all other uses, in any current or future media, including reprinting/republishing this material for advertising or promotional purposes, creating new collective works, for resale or redistribution to servers or lists, or reuse of any copyrighted component of this work in other works.

DOI 10.1109/TNET.2014.2373398

\title{
An Analytical Model for Loc/ID Mappings Caches
}

\author{
Florin Coras, Jordi Domingo-Pascual, Darrel Lewis and Albert Cabellos-Aparicio
}

\begin{abstract}
Concerns regarding the scalability of the interdomain routing have encouraged researchers to start elaborating a more robust Internet architecture. While consensus on the exact form of the solution is yet to be found, the need for a semantic decoupling of a node's location and identity is generally accepted as a promising way forward. However, this typically requires the use of caches that store temporal bindings between the two namespaces, to avoid hampering router packet forwarding speeds. In this article, we propose a methodology for an analytical analysis of cache performance that relies on the working-set theory. We first identify the conditions that network traffic must comply with for the theory to be applicable and then develop a model that predicts average cache miss rates relying on easily measurable traffic parameters. We validate the result by emulation, using real packet traces collected at the egress points of a campus and an academic network. To prove its versatility, we extend the model to consider cache polluting user traffic and observe that simple, low intensity attacks drastically reduce performance, whereby manufacturers should either overprovision router memory or implement more complex cache eviction policies.
\end{abstract}

\section{INTRODUCTION}

The growing consensus within the network community today is that a semantic separation of the identity and location information, currently overloaded in IP addresses, would solve the limitations of the routing infrastructure [1], [2]. Such architectural update involves network infrastructure upgrades in few, selected, network points like border routers, where the linking of the two namespaces must be performed. But, more importantly, it also entails the more challenging implementation of a mapping-system able to distribute dynamic locationidentity bindings (mappings) to globally distributed routers.

In this context, routers generally retrieve mappings on user demand, as opposed to proactively fetching them. This is done such that the amount of memory a router requires to participate in the system does not grow with identifier space, as is the case today, but is instead dependent on the packet level traffic the router processes. As a result, to diminish retrieval times, increase packet forwarding speed and to protect the mapping system from floods of resolution requests, routers are provisioned with mappings caches (map-caches) that temporarily store in use bindings.

Although caches placed between processor and main memory, in operating systems or in web proxies are well studied [3], [4], [5], route and mappings caches have yet to be thoroughly analyzed. A considerable number of experiments have empirically evaluated map-cache performance, however they are mainly focused on providing a circumstantial description

F. Coras, J. Domingo and A. Cabellos are with DAC, Universitat Politécnica de Catalunya, Barcelona, Spain (email: \{fcoras, acabello, jordi.domingo\}@ac.upc.edu)

D. Lewis is with Cisco Systems, San Jose, CA, USA (email: darlewis@cisco.com) of cache behavior, that is, for particular cache configurations and network traffic traces, as opposed to a general one [6], [7], [8], [9], [10]. Typically, these results yield accurate estimates for cache performance but unfortunately cannot be extrapolated to provide rough projections or bounds on cache behavior for workloads with different characteristics; nor can they provide insight into what traffic properties influence cache performance and to what degree. Answering such questions would not only be a first important step towards understanding the overall performance of the mapping-system, but would also provide a quick way of gauging the expected map-cache performance of any network domain.

In this paper, we present an analytical model that, to the best of our knowledge, constitutes the first theoretical framework for map-cache performance analysis and provisioning. The model relies on coarse traffic parameters and aims to be applicable to a wide range of scenarios. In particular, we first show how the working-set theory [11] may be used to estimate simple parameters that characterize the intrinsic locality of network traffic and thereafter explain how they can be leveraged to link cache size and miss rate. The underlying assumption that enables the analysis is that traffic can be approximated as having a stationary generating process. We find stationarity to hold for real network traffic, and, to facilitate the use of the model, we propose a simple methodology that tests for it in network traces. Finally, we validate the result by emulation, using packet traces collected at the edges of a campus and an academic network. Part of these results have been previously presented in [12].

Another contribution of this paper is that we exploit the model to $(i)$ perform an in-depth, over time analysis of cache performance for our datasets and $(i i)$ study the security of the map-cache by evaluating its vulnerability to scanning attacks. Our findings show that miss rate decreases at an accelerated pace with cache size and eventually settles to a power-law decrease. As a result, even for a relative small size, $10 \%$ of the Internet's aggregated routing table, the performance is still acceptable, below $0.2 \%$ packet miss-rate. On the other hand, we also find that even simple and low intensity attacks may compromise performance and that increasing the cache size does not alleviate the problem while the map-cache cannot accommodate the whole destination address space. Thereby, to achieve acceptable forwarding speeds, manufacturers should either overprovision router memory or design efficient cache management algorithms.

For the sake of clarity, we focus our analysis on the performance of LISP map-cache (see Section II). Nevertheless, the results are relevant for other architectures inspired by the location/identity split paradigm, including those like ILNP [13] that use DNS as their mapping system, since the equations could be used to approximate DNS resolver caching perfor- 
mance. Moreover, the cache models could be applied to routecaching and scalability techniques that focus on shrinking routing tables to extend router lifetimes [14].

The rest of the paper is structured as follows. Section II provides an overview of the location/identity split paradigm and one of its most successful implementations, LISP. In Section III we first introduce the cache model problem and then present our analytical model and its extension that accommodates for cache pollution attacks. Section IV describes the methodology used and the results that validate the two models. Section V discusses the predictions of our results when applied to our datasets and some design guidelines to improve cache performance. Section VI presents the related work. Finally, Section VII concludes the paper.

\section{LOCATION/IDENTITY SPLIT BACKGROUND}

Prompted by the growing BGP routing tables and associated churn, researchers in both academia and operational community have spent recent years (arguably the last couple of decades) trying to improve the scalability of the Internet's architecture. The factors supporting the routing table's growth are partly organic in nature, as new domains are continuously added to the topology however, they are also closely related to current operational practices [2]. In this sense, multihoming, traffic engineering and allocations of provider-independent prefixes are the main drivers behind the prefix de-aggregation that drives the increase of the routing tables. On the one hand, this effect is detrimental because it leads to engineering limitations, as the growth rate of the routing table surpasses that of its supporting technology, but it is also harmful to network operators' business since the added capital expenses result in less cost-effective networks [2].

It has been long hinted at and today it is generally accepted that the origin of this architectural inflexibility can be tracked down to the semantic overloading of the IP addresses with both location and identity information [15]. Therefore, their separation, typically referred to as a loc/id split, has been proposed by many solution that aim to mitigate the routing problems [16] but also by those aiming to integrate new features [17]. Apart from infrastructure upgrades, these architectures also require the deployment of a mapping-system for linking the two new namespaces and often rely on caches to limit router memory requirements and improve forwarding speeds.

Given that the loc/id split paradigm only outlines a set of principles, for completeness, in what follows we use the Locator/ID Separation Protocol (LISP) [18], [19], to illustrate the operation of a fully-fledged loc/id architecture. LISP is one of the most successful loc/id split implementations to date and counts with the support of a sizable community [20] dedicated to its development.

Prior to forwarding a host generated packet (see Fig. 1), a LISP router maps the destination address, an end-host identifier (EID), to a corresponding destination routing locator (RLOC). This is achieved by first looking up the destination in the local mappings cache, the map-cache, and, if this fails, by requesting the mapping to a mapping system [21], [9], [22]. So, instead of proactively storing bindings for all the possible

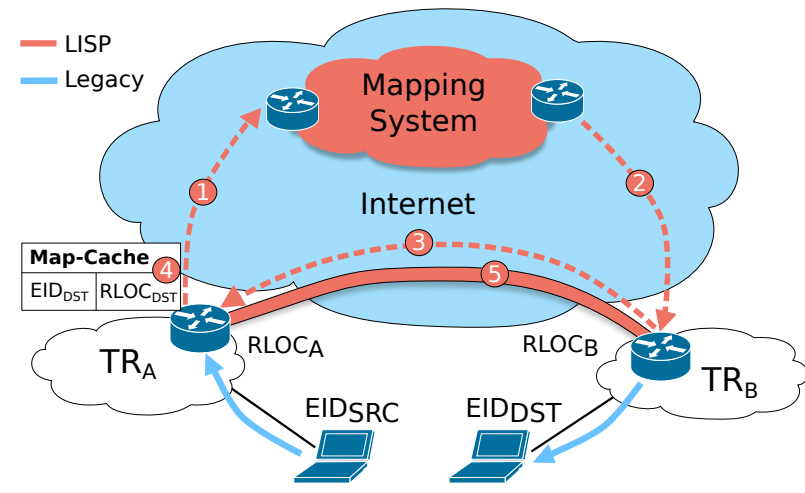

Fig. 1. Example packet exchange between $E I D_{S R C}$ and $E I D_{D S T}$ with LISP. Following intra-domain routing, packets reach $T R_{A}$ which, not having a prior map-cache entry, obtains a mapping binding $E I D_{D S T}$ to $R L O C_{B}$ from the mapping-system (steps 1-3). $T R_{A}$ stores the mapping in the mapcache (step 4) and then encapsulates and forwards the packet to $R L O C_{B}$ over the Internet's core (step 5). $T R_{B}$ decapsulates the packets and forwards them to their intended destination.

identifiers in the Internet, LISP routers request and store them in accordance to the packet level traffic they process. This ensures that map-cache size is independent of the identity namespace size and only dependent on local user needs. Once a mapping is obtained, the border router encapsulates and tunnels [23] the packet from source edge to corresponding destination edge tunnel router (TR), where the packet is decapsulated and forwarded to its intended destination.

Stale map-cache entries are avoided with the help of timeouts, called time to live (TTL), that mappings carry as attributes, while consistency is ensured by proactive LISP mechanisms which allow the owner of an updated mapping to inform its peers of the change.

The map-cache is most efficient in situations when destination EIDs present high temporal locality and its size depends on the visited destinations set size. As a result, performance depends entirely on map-cache provisioned size, traffic characteristics and the eviction policy set in place. We dedicate the remainder of the paper to finding and evaluating the relation between parameters like cache size, performance and locality of traffic.

\section{CACHe Performance}

This section presents the theoretical background and analytical methodology used to model map-cache performance. After briefly introducing the working-set theory we formalize the cache modeling problem and show that the working-set is suitable for the analysis of our network traces. The results enable us to derive a cache model and by extension one that accounts for cache polluting attacks.

\section{A. Problem Definition}

For operating systems, a general resource allocation treatment was possible after it was observed that programs often obey the so called principle of locality. The property arises from the empirical observation that programs favor only a subset of their information at a given time, so they may be efficiently run only with a fraction of their total data and 


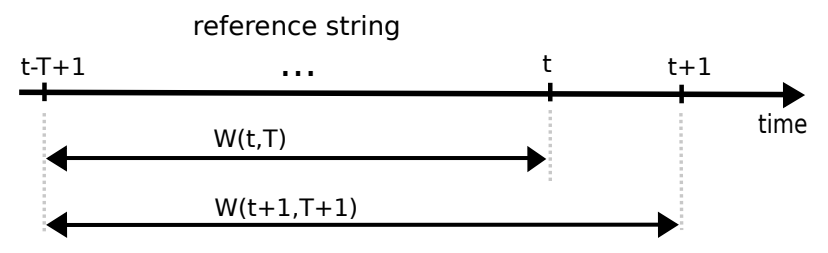

Fig. 2. The Working-Set Model. At time $t, W(t, T)$ is the set of units recently referenced in the time window $T$, i.e., during the interval $[t-T+1, t]$.

instruction code. It was shown that if the subset, called the program's locality, can be computed, the policy of keeping the locality in memory is an optimal or near optimal memory management policy [24].

Based on previous results [6], [8], [7], we argue that prefixlevel network traffic roughly obeys the same principle because of, among others, skewed destination popularity distributions and flow burstiness in time, as they gives rise to temporal locality, but also due to aggregation, i.e., multiplexing of a large number of user flows, which leads to a form of geographical locality. We therefore evaluate the feasibility of in-router prefix/mappings caching by analyzing the locality of network traffic. Since we want to avoid any assumptions regarding the structure of the process generating the network traffic, we opt in our evaluation for the working-set model of locality. For a list of other locality models see [24].

Next we provide a summary of the working-set terminology. For brevity, we use the term unit of reference, or simply unit, as a substitute for the referenced object (e.g., prefixes); and reference set to represent the set of all referenced units. Then, considering a reference set $\mathrm{N}$, we define a reference string as the sequence $\rho=r_{1} r_{2} \ldots r_{i} \ldots$ where each unit $r_{i} \in N$. If $t$ is a measure of time in units, then we can state:

Definition 1: Given a reference string, the working-set $W(t, T)$ is the set of distinct units that have been referenced among the $T$ most recent references, or in the interval $[t-T+1, t]$.

A graphical depiction can be found in Figure 2. In accordance with [25] we refer to $T$ as the window size and denote the number of distinct pages in $W(t, T)$, the working-set size, as $w(t, T)$. The average working-set size, $s(T)$, measures the growth of the working-set with respect to the size of the window $T$, extending in the past, but independent of absolute time $t$. It is defined as:

$$
s(T)=\lim _{k \rightarrow \infty} \frac{1}{k} \sum_{t=1}^{k} w(t, T)
$$

It can be proved that the the miss rate, $m(T)$, which measures the number of units of reference per unit time returning to the working-set, is the derivative of the previous function and that the sign inverted slope of the miss rate function, the second slope of $s(T)$, represents the average interreference distance density function, $f(T)$. For a broader scope discussion of these properties and complete proofs, the interested reader is referred to [25].

It is important to note that $s(T)$ and $m(T)$, if computable, provide estimates on the minimum average size of a cache able to hold the working-set, i.e., the prefixes in the active locality, and its corresponding miss rate with respect to the number of references processed. Our goal in this paper is to determine if $m(s)$ exists for real network traffic and if it can be modeled as simple function without a considerable loss of precision.

\section{B. Network Traffic Locality}

As explained by Denning in [25], a working-set analysis of reference strings may be performed only if based on three constraints that provide for a more rigorous definition for locality of reference:

1) Reference strings are unending

2) The stochastic mechanism underlying the generation of a reference string is stationary, i.e. independent of absolute time origin.

3) For $t>0, r_{t}$ and $r_{t+x}$ become uncorrelated as $x \rightarrow \infty$

The first, though obviously not fulfillable, introduces an insignificant error because the reference strings generated by practical programs or traces are long from a statistical standpoint. The third requires that references become uncorrelated as the distance goes to infinity. This can usually be asserted as being true in practice. The most restrictive of the three is the second, which limits the analysis to a locality where all three constraints, including stationarity are satisfied.

In practice however, network traffic reference strings may consists of sequences of localities when either each is characterized by a distinct stationary processes or, alternatively, when some present nonstationarities. In such scenarios the results, like the average working-set size, are only valid within a locality, and not to be extrapolated for the whole trace. To test for this possibility and to identify the reference string segments having different generating processes in network traffic traces, we devise the simple experimental methodology that follows.

To enable our analysis, since computing the $w(t, T)$ for all acceptable integer combination of $t$ and $T$ is intractable, we define the working-set curve to be $w(t, T)$ as a function of $T$, when the past time reference of the working-set is held fixed, i.e., $t-T=c s t$. For instance, considering Figure 2, $w(t, T)$ and $w(t+1, T+1)$ are consecutive points on a working-set curve with start time $t-T$. Then, for a given trace, we compute multiple empirical destination prefix working-set curves with start times spanning one day and spaced by a fixed interval. Intuitively, one would expect that the clustering patterns of the curves should distinguish between the multitude of generating process. That is, curves with close start times should have a similar growth shape (cluster), because they follow a similar sequence of localities, whereas those separated by larger time lags should behave differently. More formally stated:

Proposition 1: The clustering of the working-set curves, under which $\forall T, w(t, T)$ is normally distributed, is equivalent to the stationarity of the process generating the reference string.

We provide a proof for the the proposition in the Appendix. In addition, we empirically confirm the result by using it to determine the stationary processes embedded in four real network traffic traces. Details regarding the traffic captures can be found in Section IV-A. 


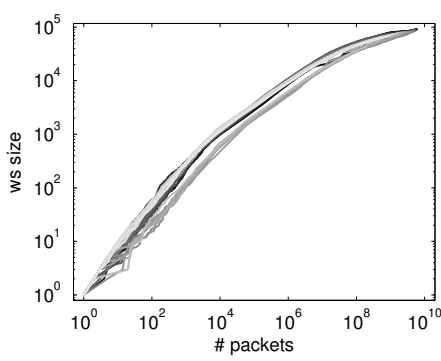

(a) upc 2009

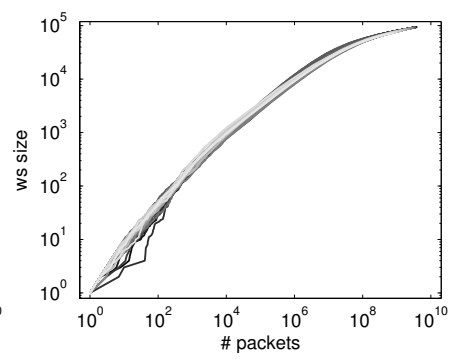

(b) upc 2011

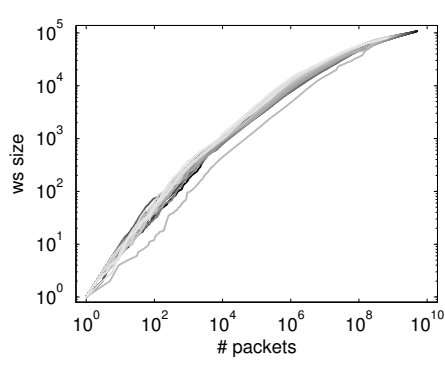

(c) $u p c 2012$

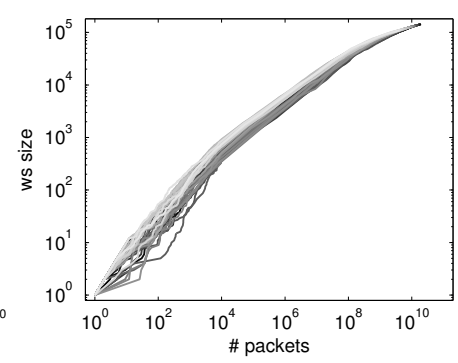

(d) cesca 2013

Fig. 3. Empirical working-set curves with starting times spaced by $30 \mathrm{~min}$ and evenly distributed over a day. Closeness of color nuance reflects closeness of start time. Notice their tendency to cluster.

For each network trace we computed working-set curves spaced by half an hour. Figure 3 presents the working-set curves of the four datasets in log-log plots. It can be noticed that all traces exhibit a strong clustering and sublinear growth, due to temporal locality. Although, the number of samples does not allow for an accurate enough testing, we could also confirm that $\forall T, w(t, T)$ is close to normally distributed by manual inspection and graphically. Therefore, in light of the previous proposition, we find that each trace can be considered as generated by a single stationary process.

To validate the result, we also independently tested the stationarity of the process generating the reference string by applying the augmented Dickey-Fuller unit root test [26] to the interreference distance time series. Due to the large size of the dataset we first aggregated the time series by computing for each window of $10 k$ data points the mean and subsequently applied the test to the resulting mean series. For all traces, the null hypothesis that the series has a unit root, with the alternative that the series is stationary, was rejected $(p<0.01)$.

Undoubtedly, the most surprising result of the analysis is the stationarity of the processes generating the four traces. It follows that the average working-set and the other metrics derived from it characterize a trace in its entirety. This might seem to be somewhat counter-intuitive if one considers the nonstationarities of network traffic when analyzed in the time domain. However, we believe stationarity to be the result of flow multiplexing, whereby the effect of short term correlations is canceled out by the influence of destination popularity, as shown in the case of Web traffic in [27].

Similarly, Kim et al. observed in [8] that the workingset size for prefixes tends to be highly stable with time for traffic pertaining to a large ISP. We would like to stress that we do not assume all traffic in the Internet is generated by stationary processes, i.e., possesses an approximate time translation invariance of the working-set curves like the one observed in Figure 3. And in fact, we require that the model we develop further be applied only to traces that have this property.

\section{Analytical Cache Model}

Because the exact form of the average working-set size, as obtained using (1), is rather cumbersome to work with, a shorter but approximate representation would be desirable.
With hindsight, one can recognize that the empirical workingset curves from Figure 3 are piecewise linear when depicted in $\log -\log$ scale. This observation enables us to approximate the average working-set size, $s(u)$, for each trace with respect to the number of packets $u$, by means of a piecewise linear fit of the $\log$ - $\log$ scale plot. We therefore obtain estimates of both the slope, $\alpha$, and the y-intercept, $\beta$, for all segments. In our results, we limited the number of segments to just four, however if better fits are desirable, more segments may be used. Through conversion to linear scale the average workingset equation becomes piecewise power law of the type:

$$
s(u)=e^{\beta(u)} u^{\alpha(u)}
$$

where, $u$ represents the number of referenced destination prefixes, or the window size, $s(u)$ is the fitted working-set size function and $\alpha(u), 0<\alpha(u) \leq 1$, and $\beta(u) \geq 0$ are piecewise constant, decreasing and respectively increasing functions obtained through fitting. Defined as such, the pair $(\alpha(u), \beta(u))$ provides a compressed characterization of the temporal locality present within a trace with respect to time, i.e., number of packets.

We can estimate the miss rate for a trace by computing the derivative of $s(u)$ like:

$$
m(u)=e^{\beta(u)} \alpha(u) u^{\alpha(u)-1}
$$

Taking the inverse of (2) and inserting it in (3) we obtain an analytical relation that links the cache size and the estimated miss rate:

$$
m(s)=e^{\beta^{*}(s) / \alpha^{*}(s)} \alpha^{*}(s) s^{1-1 / \alpha^{*}(s)}
$$

where, $s$ represents the cache size in number of entries and $\alpha^{*}(s)$ and $\beta^{*}(s)$ are piecewise constant functions with knees dependent on $s$. This equation accurately predicts cache performance over longer spans of time, if $s(u)$ is relatively stable in the considered time frame (more on this in Section V).

Regarding the type of cache modeled, it is useful to note that the working-set, $W(t, u)$, generally models a cache that always contains the $w(t, u)$ most recently referenced units. Then, given that $\forall u, w(t, u) \sim N\left(\sigma, \mu^{2}\right), W(t, u)$ actually models a cache of size normally distributed and dependent on $u$. In particular, when $\sigma$ is small, or goes to zero, it behaves like the size were fixed. Finally, because the implicit eviction policy 
requires that entries not referenced in a window of length $u$ are discarded, for low $\sigma$, like in our traces, the working-set simulates a cache of fixed size with a LRU eviction policy.

To summarize, because we find that $\forall u$, the working-set size is normally distributed and that traffic may be seen as generated by a stationary process, (4) actually models a cache with LRU eviction and size dependent on $u$. We validate the model and the efficiency of LRU in Section IV-C.

\section{Cache Model for Cache Pollution Attacks}

The model presented in the previous section can be extended to account for situations when intra-domain users perform EID space scanning attacks that significantly alter the working-set curves and therefore the shared map-cache's performance. In this work we focus on assessing the damage users can inflict through data-plane attacks and do not consider control-plane attacks like those described in [28].

We define a scanning attack as the situation when one or multiple users, acting jointly, send packets over a large period of time (e.g., hours), to destinations having a high probability of not being found in the cache. The goal would be to either generate cache misses, resulting in control plane overload or, if the cache is not large enough, to generate cache evictions, which would affect ongoing flows. For instance, having a list of EID prefixes, an attack would consist in sending packets with destinations enumerating all prefixes in the set in a random order, at a certain packet rate. Once all destinations are exhausted the enumeration would start over.

In what follows we formally define the parameters of the attack. Let, $\Omega$ be the EID-prefix set used in the attack and $\Psi$ the network's visited EID-prefix set. We define the relative attack intensity $\rho$ as the ratio between the attack packet rate and the legitimate traffic packet rate, additionally, let the attack overlap $\delta$ be the ratio between the number of prefixes common to $\Omega$ and $\Psi$ and the cardinality of $\Psi$ thus, $\delta=|\Omega \cap \Psi| /|\Psi|$.

If a network trace with average working-set $s(u)$ is augmented by a scanning attack of relative attack intensity $\rho$ and overlap $\delta$, the resulting average working-set becomes:

$$
s_{a}(u+\rho u)=\left\{\begin{array}{l}
s(u)+\rho u-\frac{\delta s\left(u_{k}\right)}{u_{k}} u, \quad u<u_{k} \\
s(u)+|\Omega|-\delta s(u)
\end{array}\right.
$$

where $u_{k}=|\Omega| / \rho$ and it represents the number of legitimate packets after which the attack exhausts all $|\Omega|$ destinations and the scan restarts. The aggregate working-set has three components. The first is due to legitimate traffic, $s(u)$, and the second, due to the attack packets, $\rho u$. However, because the two may overlap, a third component subtracts the number of shared prefixes. For simplicity, we approximate the probability of having destinations repeat to be uniform. Thereby, the growth of the overlap is linear with $u$ up to $u_{k}$, where it reaches a maximum of $\delta s\left(u_{k}\right)$ and afterwards linear with $s(u)$.

After a change of variable and denoting $\tau=1 /(1+\rho)$, or the ratio of legitimate traffic in the trace, $u_{k}=\frac{|\Omega|}{1-\tau}$ and the equation becomes:

$$
s_{a}(u)=\left\{\begin{array}{l}
s(\tau u)+\left(1-\tau-\frac{\tau \delta s\left(u_{k}\right)}{u_{k}}\right) u, u<u_{k} \\
(1-\delta) s(\tau u)+|\Omega|
\end{array}\right.
$$

Then, the miss as a function of the number of processed packets is:

$m_{a}(u)=\left\{\begin{array}{l}\tau m(\tau u)+\left(1-\tau-\frac{\tau \delta s\left(u_{k}\right)}{u_{k}}\right), u<u_{k} \\ \tau(1-\delta) m(\tau u)\end{array}\right.$

However, in this case the miss rate cannot be represented analytically as a function of the cache size since $s_{a}^{-1}(u)$ is not expressible in terms of standard mathematical functions. It can though be computed numerically as a function $u$, when $s(u)$ is known. Then, given that both $s_{a}(u)$ and $m_{a}(u)$ are known, they suffice to understand the cache's miss rate as a function of the cache size. The resulting model predicts overall cache misses, not only those due to legitimate traffic. Therefore, it provides an estimate of the control plane overload, not a data plane performance estimate for legitimate traffic. We empirically validate the result in Section IV-C.

\section{Model VAlidation}

In this section, we present the evaluation methodology and the results that validate our models. We start by describing our datasets and then pass on to present the ITR emulator employed in the empirical evaluation of the cache. Last, we compare the empirical results to those predicted by the analytical models.

\section{A. Datasets}

Four one-day packet traces were used for the purpose of our experiments. Three of them have been captured at the $2 \mathrm{Gbps}$ link connecting several of our University's campus networks to the Catalan Research Network (CESCA) and consist only of egress traffic. UPC campus has more than 36k users. The first trace dates back to May 26th, 2009 while the other two are from October 19th, 2011 and November 21st, 2012. The fourth trace was captured on January 24th, 2013 at CESCA's 10Gbps link connecting it to the Spanish academic network (RedIris) and consists of egressing academic traffic. In 2011, CESCA was providing transit services for 89 institutions, including the greater part of public Catalan schools, hospitals and universities.

Table I summarizes some of the important properties of our datasets. First of all, it can be seen that cesca 2013, being an aggregate trace, is about 3.6 times larger than the most recent UPC trace in terms of number of packets and packet rate. However, it only contains 1.3 times more prefixes. This shows that although the number of users and packets exchanged is considerably higher, the diversity of the destinations is only slightly incremented. Out of the UPC traces, upc 2009 exhibits a surprisingly high number of packets but this is explained by the very large packet rates seen during the active hours of the day. In fact, the average packet rate during the peak 


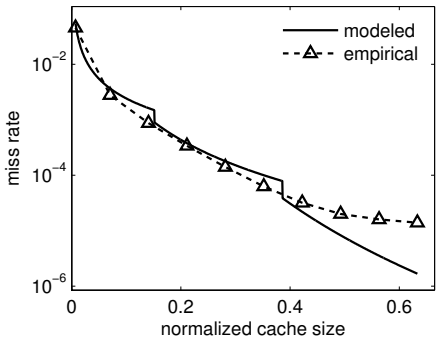

(a) upc 2009

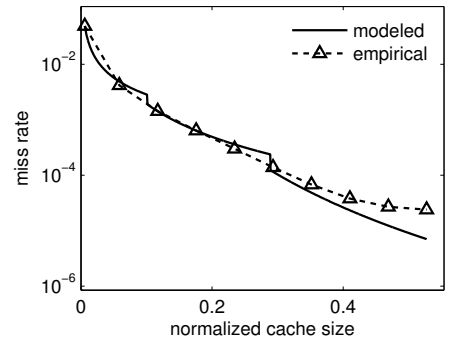

(b) upc 2011

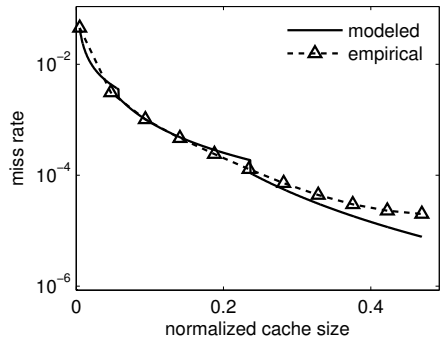

(c) upc 2012

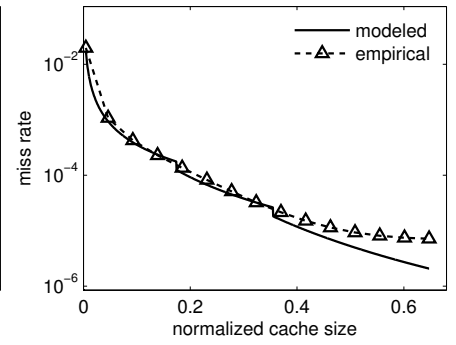

(d) cesca 2013

Fig. 4. Comparison between empirical and modeled miss rates, as estimated by (4), for normal cache operation.

TABLE I

DATASETS STATISTICS

\begin{tabular}{lcccc}
\hline & upc 2009 & upc 2011 & upc 2012 & cesca 2013 \\
\hline Date & $2009-05-26$ & $2011-10-19$ & $2012-11-21$ & $2013-01-24$ \\
\hline Packets & $6.5 \mathrm{~B}$ & $4.05 \mathrm{~B}$ & $5.57 \mathrm{~B}$ & $20 \mathrm{~B}$ \\
\hline Av. pkt/s & 75312 & 46936 & 64484 & 232063 \\
\hline$\Psi$ & 92801 & 94964 & 109451 & 143775 \\
\hline Av. pref/s & 2323 & 1952 & 2123 & 2560 \\
\hline
\end{tabular}

hours was 4.7 times higher that for the rest of the day. Again, this difference did not reflect in the number of unique prefixes observed in a one second window as, on average, we observed just 1.3 times more prefixes in the peak hours than during the remainder of the day. These two observations suggest that higher packet rates, either resulting from larger user sets or from higher throughput flows, do not increase destination diversity (as illustrated in Figure 3) but instead reinforce temporal locality. In addition, these properties also explain why the working-set curves for upc 2009 present a time-of-day behavior (see Figure 3a).

\section{B. Map-Cache Emulator}

To evaluate the two models and the effectiveness of the working-set as a tool for cache performance prediction, we implemented a packet trace based emulator that mimics basic ITR functionality.

Both for computing the working-sets in Section III-B and for the cache performance evaluation, destination IP addresses had to be mapped to their corresponding prefixes. We considered EID-prefixes to be of BGP-prefix granularity. For each traffic trace, we linked IP addresses to prefixes using BGP routing tables (BGP $R T$ ) downloaded from the RouteViews archive [29] that matched the trace's capture date. In particular, we used collector route-views4 situated at University of Oregon. The only preprocessing we performed was to filter out more specific prefixes. Generally, they are used for traffic engineering purposes but LISP provides mechanisms for a more efficient management of these operational needs that do not require EID-prefix de-aggregation. We refer to the resulting list as $\mathrm{BGP}_{\phi}$. Table II shows the size of the routing tables used for each trace and provides the proportion of prefixes seen within each trace out of the total registered in
TABLE II

ROUTING TABLES STATISTICS

\begin{tabular}{lcccc}
\hline & upc 2009 & upc 2011 & upc 2012 & cesca 2013 \\
\hline $\mathrm{BGP}_{R T}$ & 288167 & 400655 & 450796 & 455647 \\
\hline $\mathrm{BPG}_{\phi}$ & 142154 & 170638 & 213070 & 216272 \\
\hline$\Psi / \mathrm{BGP}_{\phi}$ & 0.65 & 0.55 & 0.51 & 0.66 \\
\hline
\end{tabular}

the filtered routing table, $\Psi / \mathrm{BGP}_{\phi}$. It may be seen that, as the ratio is always higher that 0.5 , more than half of the possible destination prefixes are visited in one day for all traces.

For each packet processed, the emulator maps the destination IP address to a prefix in $\mathrm{BGP}_{\phi}$. If this prefix is already stored in the ITR's cache, its cache entry is updated and the emulator continues with the next packet. Should the prefix not yet be stored in the cache, two possibilities arise. First, if the cache is not full, the destination prefix is stored in and the processing proceeds to the next packet. Second, if the cache is full, an entry is evicted, the new prefix is stored in and then the emulator moves to the next packet. The entry to be evicted is chosen according to the LRU eviction policy. We use LRU because, as mentioned in Section III-C, its performance should be close to optimal due to the stationarity of the trace generating process. Accordingly, the performance of the cache should be appropriately described by (4).

Scanning attacks are emulated by uniform insertion of attack packets in-between those pertaining to the processed traffic trace, according to the relative attack intensity $\rho$. The number of attack addresses generated depends on $\delta$, the overlap between $\Omega$ and $\Psi$, and the number of destinations in $\mathrm{BGP}_{\phi}$. Note that $\Omega \subseteq \mathrm{BGP}_{\phi}$ and $\Psi \subseteq \mathrm{BGP}_{\phi}$. Therefore, supposing the attack maximizes number of addresses used, to increase effectiveness, $|\Omega|=\left|\mathrm{BGP}_{\phi}-\Psi\right|+\delta|\Psi|$. In particular, when no overlap exists, we generate $|\Omega|=\left|\mathrm{BGP}_{\phi}-\Psi\right|$ new destination addresses while for a full overlap, the attack consists of $|\Omega|=\left|\mathrm{BGP}_{\phi}\right|$ addresses. If $\delta \neq 0$, the addresses used in the attack and part of $\Psi$ are uniformly distributed among those part of $\mathrm{BGP}_{\phi}-\Psi$.

\section{Comparison of Analytical and Empirical Results}

To validate the models we use the emulator to estimate empirical cache miss rate for several cache sizes. Figure 4 presents a comparison of the empirical and predicted results 


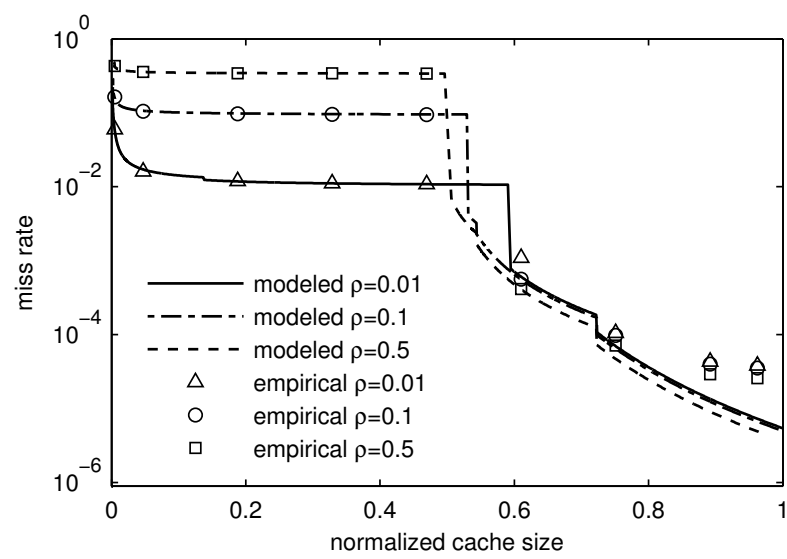

(a) No overlap $(\delta=0), \Omega=\mathrm{BGP}_{\phi}-\Psi$

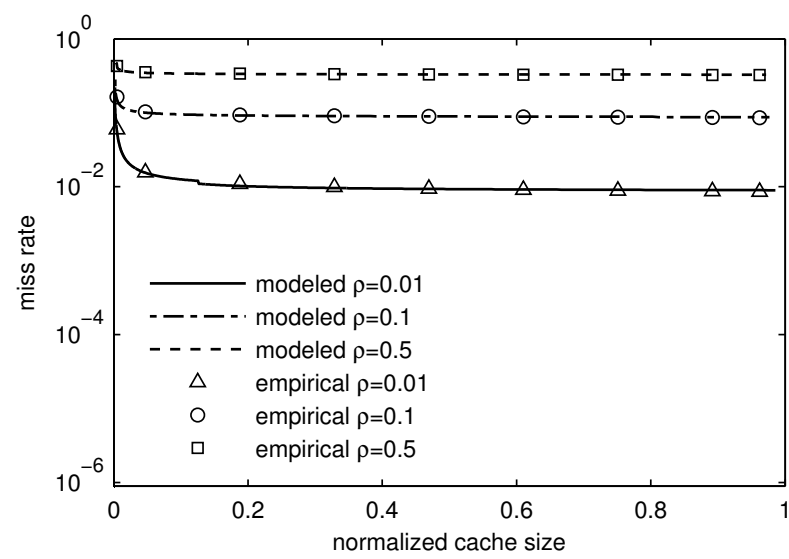

(b) Complete overalp $(\delta=1), \Omega=\mathrm{BGP}_{\phi}$

Fig. 5. Comparison between empirical and modeled miss rates, as estimated by (6) and (7), under scanning attacks with attack intensity $\rho \in\{0.01,0.1,0.5\}$ for upc 2012.

for normal traffic, where cache size is normalized with corresponding $\mathrm{BGP}_{\phi}$ routing table size (see Table II). It may be seen that, typically, the absolute error is negligible despite the discontinuities of the model, which are due to the piecewise fitting. Further, the equation appropriately predicts that performance stays acceptable, even for small cache sizes, fact also observed by [6], [7], [9]. The result is even more remarkable as we never remove stale entries in our emulator, i.e., we consider TTL to be infinite.

The figures present the cache miss rate only up to a fraction of $\mathrm{BGP}_{\phi}$, the one associated to $|\Psi|$, because the growth of $s(u)$ cannot be extrapolated after this point. Since the working-set grows slower for larger $u$, as $\alpha(u)$ is a strictly decreasing function, potentially much longer traces would be needed to enable inference about larger cache sizes. In fact, given that only part of the whole prefix space may be visited by the clients of a stub network, even for longer traces the analysis may be limited to a cache size lower than $\left|\mathrm{BGP}_{\phi}\right|$.

There are two limitations to the precision of our analysis for large $u$ values. First, as cache size increases and approaches $|\Psi|$, the accuracy of the prediction diminishes. This is explained by the 24-hour length of the traces, whereby there are few working-set curves that span close to a whole day and thus grow to reach the maximum number of destination prefixes. Recall that the start times for the working-set curves span the whole trace and are spaced by 30 minutes, so the last curves consist of few packets. As a consequence, $s(u)$ is estimated using a reduced number of points, i.e., with a lower precision, at the higher end. To counter this effect, we compute average working-sets of slightly diminished length. The second limitation is the bias of our emulation results for large cache sizes. Caches whose sizes are close to $|\Psi|$ fill only once the traces are processed almost in their entirety. Due to this cold-start effect, cache are exposed to a low number of hits up to the end of the traces. As these hits do not manage to outweigh the misses generated during the cache fill up, the miss rate of the emulator for large cache sizes is slightly overestimated. Nevertheless, despite these limitations, it may be seen that the results still yield a good fit for large $u$.
We also validate our cache model that accounts for scanning attacks considering as scenarios a complete attack overlap or one that is zero. Thereby, the attackers may use as attack prefix set either the whole EID-prefix space or just the part not visited by the attacked network's clients. In the latter case, if the cache is not large enough to hold all prefixes, all attack packets would generate a cache miss. However, note that building such a prefix set would require full knowledge about the network's traffic. In the former case, some packet destinations may generate cache hits but $|\Omega|$ may potentially be much larger and this could prove beneficial to the attacker. In light of these properties we consider the two attacks as worst case scenarios, from the attacked network's perspective, for the situations when attackers respectively have or do not have knowledge about the attacked network's traffic.

Figure 5 compares the analytical and empirical results for the cache miss rate, when $\delta \in\{0,1\}$ and $\rho \in\{0.01,0.1,0.5\}$. We present the results just for upc 2012 since those for the other three traces are similar. It may be observed that for both no overlap and complete overlap the results exhibit little absolute error. In particular, for $\delta=0$ when cache size is larger and miss rates are less than $10^{-4}$ the errors are more significant. As in the case of the cache model, this is explained by the trace length and the reduced number of points used in estimating the higher end of $s(u)$. The effect is not noticeable for $\delta=1$ because $s_{a}(u)$ reaches its maximum (saturates) for low values of $u$ due to the larger attack set. Consequently, the fit is very good along the whole spectrum of cache sizes. Once the cache size reaches $\left|\mathrm{BGP}_{\phi}\right|$ the miss rate becomes 0 since there are no more destination prefixes outside those already present in the cache to generate a miss.

\section{Discussion}

In this section we discuss the results and predictions of our models regarding map-cache performance for both normal and malicious traffic. We also discuss possible avenues to diminishing the effect of cache attacks.

The results we obtained are relevant only when reasoned about jointly with the traffic traces used in the analysis. 
However, the diversity of our data sets and previous results from Kim [8], Iannone [6] and Kim [7] suggest that the properties uncovered are not the expression of isolated user and network behavior.

\section{A. Cache Model Results and Predictions}

Under the condition of a stationary generating process or, equivalently, the approximate time translation invariance of the working-set curves, our methodology enables the estimation of the time invariant piecewise functions $\alpha(u)$ and $\beta(u)$ that characterize the locality of a network traffic trace from the average working-set size $s(u)$. This further facilitates the following two findings. First, due to the low variance of $s(u)$ and experimentally proven good performance, we can now recommend the use of the LRU eviction policy for LISP caches. Second, in such situation, (4) may be used to dimension the cache sizes in operational environments, according to the desired miss rate. The prediction of its mathematical expression, considering that $\alpha^{*}(c) \rightarrow 0$ when $c$ increases, is that miss rate decreases at an accelerated pace with cache size and finally settles to a power-law decrease. This may also be observed in Figure 4 where at each discontinuity point the function switches to a faster decreasing curve. Of course, the speed of the decrease depends on the degree of locality present in the trace. Overall the equation indicates that cache sizes need not be very large for obtaining good performance. For instance, having a cache of size $10 \%$ of $B G P_{\phi}$, about $14 k-21 k$ entries for UPC traces and $21 k$ for the CESCA one, would result in a miss rate of approximately $0.09 \%-0.2 \%$ and respectively $0.03 \%$.

In this context, an important point would be to determine the extent of time over which the results and predictions hold. Figure 6 provides a coarse answer for the particular case of the traffic used in our analysis. First, considering the UPC traces, it may be observed that over a span of tree and a half years, the average working-set is rather stable when size is less than about $50 k$ prefixes. In fact, the $s(u)$ curves of upc 2011 and upc 2012 are very similar, independent of $u$ value, while the one for upc 2009 exhibits a lower slope for $u>100 M$ packets. This might appear to be in agreement with the relative, year-over-year, increase of $B G P_{\phi}$. But the relative differences between the values of $s(u)$ and the increase of $B G P_{\phi}$ are not directly related since the growth from 2009 to 2011 was smaller than the one from 2011 to 2012 . This is to be expected because many of the new prefixes, resulting from the sustained growth of the Internet's edge [30], may never become destinations for users of other edge networks. So, a direct relation between the increment of the routing table size and that of $s(u)$ should not necessarily be expected. Instead, given the good overlap for lower values of $u$, prefix popularity distribution should be more relevant to the shape of the working-set than the absolute number of destination. Second, the comparison between cesca 2013 and the UPC traces reveals that larger user sets, and implicitly higher traffic rates, result in a slightly slower growing $s(u)$. In fact, the only noticeable difference is at short time scales, where the larger trace has a smaller slope. This could be explained by a

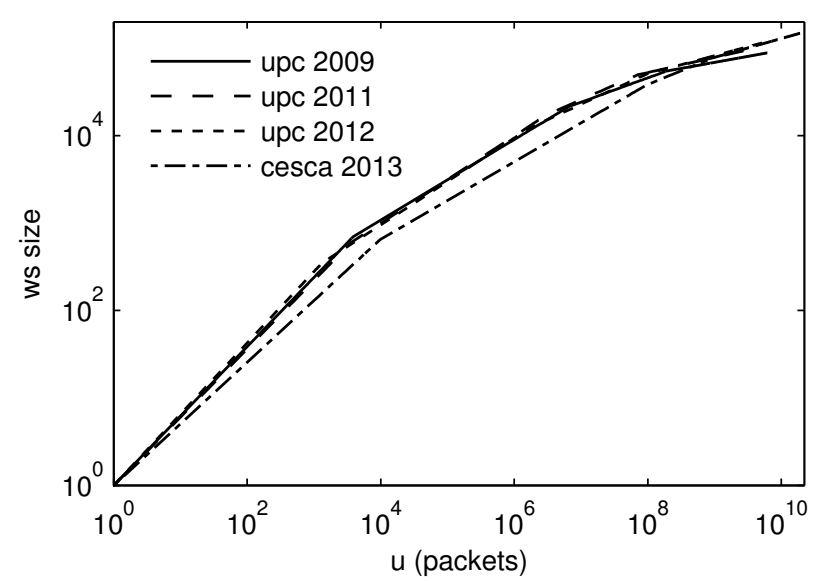

Fig. 6. Evolution of $s(u)$ over the years. The shape appears to be influenced by the number of users and the destination popularity distribution.

change in the destination popularity distribution, as cesca 2013 aggregates more types of user profiles, but also by a shift in the short-term temporal correlation. However, considering the large number of users, their synchronization at short time scales seems rather unlikely.

Then, although apparently stable over relatively long time spans, the shape of $s(u)$ seems to be influenced by non trivial interactions between the number of clients the prefix popularity distribution and possibly other unexplored factors. We are not ready to model these interactions here. Thus, our cautious inference is that the average working-set should be stable over time, if the number of clients and the popularity distribution are relatively stable.

Despite not being indicated by our measurements, it may be finally proven that the variability with time of $s(u)$ is highly dependent on properties of the network being measured, themselves time dependent. Should this be the case, the methodology we develop in this paper is still valuable for the analysis of cache performance if not for long term provisioning of caches.

\section{B. Cache Poisoning and Management Strategies}

We compared the effect of scanning attacks with zero or complete overlap to identify the one most damaging and to quantify their effect on cache performance. Associated results were presented in Figure 5.

If cache sizes are small, both attacks results in very high cache miss rate, including for $\rho=0.01$, when the attack has a rate of only 644 packets per second. In this range, miss rate is almost independent of attack overlap, only slightly higher for $\delta=0$ due to the informed selection of attack address space. However, for $\delta=0$ the cache performance is much improved after a certain threshold is passed whereas for $\delta=1$ it barely changes up to when cache size becomes $\mathrm{BGP}_{\phi}$ and miss rate drops to 0 . In other words, the non overlapping attack may be absorbed with larger cache sizes while the overlapping one no. Perhaps counter-intuitively, overlapping attacks are more damaging against a map-cache. They are easier to generate, as they do not require prior knowledge about the attacked 
network. But, they are also harder to defend against since, after a certain point and for a wide ranges of values, increasing the cache sizes does not yield much improved performance if less than $\mathrm{BGP}_{\phi}$.

Arguably, the most worrisome result we observe is the rather high miss rate which barely drops under 0.01 , even for $\rho=$ 0.01 and only when the normalized cache size is higher than 0.2 . As a comparison, under normal operation this miss rate would be obtained with a normalized cache size of about 0.02 , an order of magnitude less. Therefore, considering the high packet throughput of border routers, some more complex cache management strategies should be set in place to avoid hundreds to thousands of packet drops per second.

One possible first step to circumventing the effect of cache polluting attacks would be to detect them prior to taking action. This may be achieved with $s(u)$, if a ground truth estimation of its shape exists. So, if $s(u)$ is known beforehand, an estimate of the average miss rate for the cache size used can be computed. Then, if the instantaneous miss rate surpasses this estimate by more than configured threshold, a cache protecting action could be taken. For instance, the top, or most recently used, part of the cache could be "protected" against eviction. Other measures could include the implementation of a cache hierarchy or the limiting of user request rate for new destinations. In the former case, evicted entries should be stored in a larger but higher access time cache while in the latter some of the network elements should monitor per user traffic and filter out attack attempts. Since it is not within the scope of this paper we do not explore other aspects related to the implementation of such tools.

\section{RELATED WORK}

Feldmeier [31] and Jain [32] were among the first to evaluate the possibility of performing destination address caching by exploiting the locality of traffic in network environments. Feldmeier analyzed traffic on a gateway router and showed that caching of both flat and prefix addresses can significantly reduce routing table lookup times. Jain however performed his analysis in an Ethernet environment. He was forced to concede that despite the locality observed in the traffic, small cache performance was struggling due to traffic generated by protocols with deterministic behavior. Both works were fundamental to the field however their results were empirical and date back to the beginning of the 1990s, years when the Internet was still in its infancy.

Recently, Kim et al. [8] showed the feasibility of route caching after performing a measurement study within the operational confinement of an ISP's network. They show by means of an experimental evaluation that Least Recently Used (LRU) cache eviction policy performs close to optimal and that working-set size is generally stable with time. We also observe the stability of the working-set for our data sets but we further leverage it to build a LRU model instead of just empirically evaluating its performance.

Several works have previously looked at cache performance in location/identity split scenarios considering LISP as a reference implementation. Iannone et al. [6] performed an initial trace driven study of the LISP map-cache performance. Instead of limiting the cache size by using an eviction policy, their cache implementation evicted stale entries after a configurable timeout value. Further, Kim et al. [7] have both extended and confirmed the previous results with the help of a larger, ISP trace and by considering LISP security aspects in their evaluation. Ignoring control plane security concerns, which we did not consider, and despite differences regarding the cache eviction policy, the results of these last two works seem to be in agreement with ours. Zhang et al. [10] performed a trace based mappings cache performance analysis assuming a LRU eviction policy. They used two 24-hour traffic traces captured at two egressing links of the China Education and Research Network backbone network. They concluded that a small cache can offer good results. Finally, Jakab et al. [9] analyzed the performance of several LISP mapping systems and, without focusing on a cache analysis, also observed very low miss rates for a cache model similar to that used in [6].

Our work confirms previous LISP cache analysis results however, it also tries to provide a better understanding of the reasons behind the relatively good performance of map-caches. In this sense it introduces an analytical model that could be used to theoretically evaluate or dimension for operational needs the caching performance. Moreover, to the best of our knowledge, it is also the first work to perform an analysis and propose an analytical model for the map-cache performance when under scanning data-plane attacks.

\section{CONCLUSIONS}

The implementation of a location/identity split at network level has been recently recommended as a viable solution to the Internet's routing scalability problems. But, unless deployed with care, the cure may prove worse than the disease. Hence, a good understanding of the newly introduced components, like the map-cache, is paramount.

In this paper, we propose a methodology to evaluate mapcache performance. Our model is built by exploring the link between cache performance and parameters that approximate the intrinsic locality of packet level user traffic. To this end, we advance the use of the working-set model as a tool to capture said properties but also as a performance predictor. Accordingly, we define a framework wherein to perform the analysis and find that the clustering of the working-set curves is the only condition needed to ensure the accuracy of the model. We empirically validate our result by emulation, using traffic traces collected at the edges of a campus and an academic network.

Besides the possibility of using the model for cache dimensioning or detecting attacks in operational environments, we believe the equation may also be used as part of more complex models that evaluate the scalability of loc/id architectures. To stress these points, we show the versatility of our methodology by characterizing map-cache performance for our datasets and by building an extension that accounts for cache pollution attacks. Our observations indicate that increasing cache size quickly diminishes miss rates in normal conditions but has little to no effect under simple cache pollution attacks. In 
the latter case, we advise that more complex management strategies be devised and set in place. In the future, we plan to fully investigate the sources of locality in prefix level network traffic to better understand their impact on cache performance.

\section{ACKNOWLEDGEMENTS}

We want to express our gratitude to Damien Saucez, Luigi Iannone and Pere Barlet for their insightful comments. This work has been partially supported by the Spanish Ministry of Education under scholarship AP2009-3790, research project TEC2011-29700-C02, Catalan Government under project 2009SGR-1140 and a Cisco URP Grant.

\section{REFERENCES}

[1] G. Huston, "BGP Report." [Online]. Available: http://bgp.potaroo.net/

[2] D. Meyer, L. Zhang, and K. Fall, "Report from the IAB Workshop on Routing and Addressing," RFC 4984 (Informational), Internet Engineering Task Force, Sep. 2007.

[3] A. Agarwal, J. Hennessy, and M. Horowitz, "An analytical cache model," ACM Trans. Comput. Syst., vol. 7, no. 2, pp. 184-215, May 1989.

[4] L. Breslau, P. Cao, L. Fan, G. Phillips, and S. Shenker, "Web caching and zipf-like distributions: evidence and implications," in INFOCOM '99, Proceedings. IEEE, vol. 1, 1999, pp. 126-134 vol.1.

[5] L. Rizzo and L. Vicisano, "Replacement policies for a proxy cache," IEEE/ACM Trans. Netw., vol. 8, no. 2, pp. 158-170, Apr. 2000.

[6] L. Iannone and O. Bonaventure, "On the Cost of Caching Locator/ID Mappings," in Proceedings of the 3rd International Conference on emerging Networking EXperiments and Technologies (CoNEXT'07). ACM, Dec. 2007, pp. 1-12.

[7] J. Kim, L. Iannone, and A. Feldmann, "A deep dive into the LISP cache and what ISPs should know about it," in Proceedings of the 10th international IFIP TC 6 conference on Networking - Volume Part I, ser. NETWORKING'11, 2011, pp. 367-378.

[8] C. Kim, M. Caesar, A. Gerber, and J. Rexford, "Revisiting Route Caching: The World Should Be Flat," in Proceedings of the 10th International Conference on Passive and Active Network Measurement, ser. PAM '09, 2009, pp. 3-12.

[9] L. Jakab, A. Cabellos-Aparicio, F. Coras, D. Saucez, and O. Bonaventure, "LISP-TREE: A DNS Hierarchy to Support the LISP Mapping System," Selected Areas in Communications, IEEE Journal on, vol. 28 no. 8, pp. $1332-1343$, october 2010.

[10] H. Zhang, M. Chen, and Y. Zhu, "Evaluating the performance on ID/Loc mapping," in Global Telecommunications Conference (GLOBECOM 2008), 2008, pp. 1-5.

[11] P. J. Denning, "The working set model for program behavior," Commun. $A C M$, vol. 11 , no. 5, pp. 323-333, 1968.

[12] F. Coras, A. Cabellos-Aparicio, and J. Domingo-Pascual, "An analytical model for the LISP cache size," in NETWORKING 2012. Springer Berlin Heidelberg, 2012, pp. 409-420.

[13] R. Atkinson and S. Bhatti, "Identifier-Locator Network Protocol (ILNP) Architectural Description," RFC 6740 (Experimental), Internet Engineering Task Force, Nov. 2012. [Online]. Available: http://www.ietf.org/rfc/rfc6740.txt

[14] H. Ballani, P. Francis, T. Cao, and J. Wang, "Making routers last longer with viaggre." in NSDI, vol. 9, 2009, pp. 453-466.

[15] J. Saltzer, "On the Naming and Binding of Network Destinations," RFC 1498 (Informational), Internet Engineering Task Force, Aug. 1993. [Online]. Available: http://www.ietf.org/rfc/rfc1498.txt

[16] T. Li, "Recommendation for a Routing Architecture," RFC 6115 (Informational), Internet Engineering Task Force, Feb. 2011.

[17] X. Misseri, J.-L. Rougier, and D. Saucez, "Internet routing diversity for stub networks with a map-and-encap scheme," in Communications (ICC), 2012 IEEE International Conference on. IEEE, 2012, pp. 28612866.

[18] D. Farinacci, V. Fuller, D. Meyer, and D. Lewis, "The Locator/ID Separation Protocol (LISP)," RFC 6830 (Experimental), Internet Engineering Task Force, Jan. 2013. [Online]. Available: http://www.ietf.org/rfc/rfc6830.txt

[19] D. Saucez, L. Iannone, O. Bonaventure, and D. Farinacci, "Designing a Deployable Internet: The Locator/Identifier Separation Protocol," IEEE Internet Computing, vol. 16, pp. 14-21, 2012.
[20] "LISP Testbed." [Online]. Available: http://www.lisp4.net/

[21] V. Fuller, D. Farinacci, and D. Lewis, "LISP Delegated Database Tree (LISP-DDT)," draft-ietf-lisp-ddt-00, Internet Engineering Task Force, Nov. 2011, work in progress.

[22] M. Menth, M. Hartmann, and M. Hofling, "FIRMS: a future Internet mapping system," Selected Areas in Communications, IEEE Journal on, vol. 28, no. 8, pp. 1326-1331, 2010.

[23] R. Hinden, "New Scheme for Internet Routing and Addressing (ENCAPS) for IPNG," RFC 1955 (Informational), Internet Engineering Task Force, Jun. 1996

[24] J. R. Spirn and P. J. Denning, "Experiments with program locality," in Proceedings of the December 5-7, 1972, fall joint computer conference, part I. ACM, 1972, pp. 611-621.

[25] P. J. Denning and S. C. Schwartz, "Properties of the working-set model," Commun. ACM, vol. 15, no. 3, pp. 191-198, 1972.

[26] A. Banerjee, J. Dolado, J. Galbraith, and D. F. Hendry, "Cointegration, error correction and the econometric analysis of non stationary data."

[27] S. Jin and A. Bestavros, "Sources and characteristics of web temporal locality," in Modeling, Analysis and Simulation of Computer and Telecommunication Systems, In Proceedings. International Symposium on. IEEE, 2000, pp. 28-35.

[28] D. Saucez, L. Iannone, and O. Bonaventure, "LISP Threats Analysis," draft-ietf-lisp-threats-04.txt, Internet Engineering Task Force, Feb. 2013, work in progress.

[29] University of Oregon, "RouteViews Project." [Online]. Available: http://www.routeviews.org

[30] L. Cittadini, W. Muhlbauer, S. Uhlig, R. Bush, P. Francois, and O. Maennel, "Evolution of internet address space deaggregation: myths and reality," Selected Areas in Communications, IEEE Journal on, vol. 28, no. 8, pp. 1238-1249, 2010.

[31] D. Feldmeier, "Improving gateway performance with a routing-table cache," in INFOCOM'88. Networks: Evolution or Revolution, Proceedings. Seventh Annual Joint Conference of the IEEE Computer and Communcations Societies, IEEE. IEEE, 1988, pp. 298-307.

[32] R. Jain, "Characteristics of destination address locality in computer networks: A comparison of caching schemes," Computer networks and ISDN systems, vol. 18, no. 4, pp. 243-254, 1990.

\section{APPENDIX}

Proof: If $\forall T$ the working-set size $w(t, T)$ is normally distributed, and therefore independent of $t$, it follows that the process generating $w(t, T)$ is stationary. This in turn implies the stationarity of the process generating the reference string and, as a result, necessity is proven. It remains to be proved that if the working-set curves are generated by the same stationary process then they will tend to cluster. In [25] it is shown that for a certain window size $T$ the distribution of $w(t, T)$ converges to a normal distribution if the locality conditions hold. This proves sufficiency. 